Supporting Information For

\title{
Large-scale Spinning of Silver Nanofibers as Flexible and Reliable Conductors
}

Ya Huang ${ }^{1}$, Xiaopeng Bai ${ }^{1}$, Ming Zhou ${ }^{2}$, Suiyang Liao ${ }^{1}$, Zongfu $\mathrm{Yu}^{2}$, Yaping $\mathrm{Wang}^{3}$ and Hui $\mathrm{Wu}^{1, *}$

${ }^{1}$ State Key Laboratory of New Ceramics and Fine Processing, School of Materials Science and Engineering, Tsinghua University, Beijing 100084, China. ${ }^{2}$ Department of Electrical and Computer Engineering, University of Wisconsin, Madison, WI, USA. ${ }^{3}$ Suzhou InReal Optoelectronics Material Technology Co., Suzhou 215000, China.

E-mail: huiwu@tsinghua.edu.cn

This file includes:

Materials and Methods

Supplementary Text

Figs. S1 to S12

Video $\mathrm{S} 1$ to $\mathrm{S} 2$ 


\section{Materials and methods}

\section{Chemicals.}

Silver nitrate (99.8\%, Beijing Chemical Works), poly vinylpyrrolidone (PVP, $\mathrm{Mw}=1300000$, Alfa Aesar), acetonitrile (99.0 \%) were purchased from Xilong Scientific company and used without any purification.

\section{Preparation of silver nanofibers.}

In a typical experiment, $0.2 \mathrm{~g}$ of PVP was dissolved into acetonitrile, and then $1 \mathrm{~g}$ of silver nitrate was added and dissolved in the solution to form $2 \mathrm{~mL}$ of transparent mixed solution. The mixture solution was loaded into a $1 \mathrm{~mL}$ syringe with the specific coaxial needle. In general, the precursor solution with $10 \mathrm{wt} \%$ PVP was injected from the axle with the speed of $0.75 \mathrm{~mL} / \mathrm{h}$, the collected distance was $20-50 \mathrm{~cm}$. The fibers obtained was calcinated at $280{ }^{\circ} \mathrm{C}$ with the heating rate $5{ }^{\circ} \mathrm{C} / \mathrm{min}$ for $120 \mathrm{~min}$ and cooling with the furnace. Then the PVP were oxidized and $\mathrm{AgNO}_{3}$ was reduced to silver crystal and we can get silver nanofibers.

\section{Preparation of silver nanofiber sponge.}

Precursor of PVP/AgNO 3 fiber networks were first calcinated at $250{ }^{\circ} \mathrm{C}$ for $2 \mathrm{~h}$ before entangle treatment. Then the primary silver sponge were maintain at $280{ }^{\circ} \mathrm{C}$ for $2 \mathrm{~h}$ to fuse the junction.

\section{Physical characterization.}


The microstructure of the silver fibers was characterized by field-emission scanning electron microscopy (FESEM, LEO-1530, Zeiss, Germany) and Transmission Electron Microscope (TEM, JEOL-2010). Transparence spectra were collected using an UV-Vis-NIR spectroscope (Lambda 950, Perkin-Elmer, America) with bare quartz glass as the reference in the range of 400 to $800 \mathrm{~nm}$, and the effect of the reference was subtracted from the data published. The square resistance was measured by a ST-2258C multifunction digital four-point probe tester. The values we reported are the average value of many measurement across the entire film, providing a more accurate presentation of the conductivity. The crystal structure was recorded using X-ray diffraction (XRD, D/max-2500, Rigaku) with $\mathrm{Cu} \mathrm{K} \alpha$ radiation at a scanning rate of $5 \%$ min or $0.02^{\circ} / \mathrm{min}$ in $2 \theta$ ranging from $30^{\circ}$ to $80^{\circ}$. X-ray photoelectron spectroscopy measurements for silver fibers were performed using X-ray photoelectron spectrometer ( Escalab 250Xi, Thermo Fisher) equipped with an $\mathrm{Al} \mathrm{K} \alpha$ radiation source $(1487.6 \mathrm{eV})$ and hemispherical analyzer with a pass energy of $30.0 \mathrm{eV}$ and an energy step size of $0.05 \mathrm{eV}$. Raman spectra were measured with a Raman spectrometer (LabRAM HR Evolution, HORIBA Jobin Yvon, French). The wavelength of Raman laser is $532 \mathrm{~nm}$, and power of laser is about $750 \mathrm{~W}$. 


\section{Supplementary Text}

In order to determine the optimum temperature of calcination, we tested the Thermal Gravity Analysis (TGA) and Differential Scanning Calorimetry (DSC) of $\mathrm{PVP} / \mathrm{AgNO}_{3}$ fibers from 50 to $500{ }^{\circ} \mathrm{C}$ (Figure S1). From the TGA result, the mass of the fiber decreased steeply from 200 to $250{ }^{\circ} \mathrm{C}$ and nearly stable above $350{ }^{\circ} \mathrm{C}$. From the DSC and TGA result, there are two decomposition stage from $200{ }^{\circ} \mathrm{C}$ to $400{ }^{\circ} \mathrm{C}$. After calcination at $280{ }^{\circ} \mathrm{C}$, there is still residual carbon in the silver nanofibers which is beneficial for the flexibility. As shown in Figure S2, the $\mathrm{PVP} / \mathrm{AgNO}_{3}$ fiber before calcination has only a Raman active modes at $1039 \mathrm{~cm}^{-1}$, attributing to $\mathrm{NO}_{3}{ }^{-}$ion. While there are two Raman active mode at 1352 and $1560 \mathrm{~cm}^{-1}$ which is corresponding to amorphous carbon after the fiber was calcinated. For the silver fibers calcinated at 300 for $2 \mathrm{~h}$, the intensity of amorphous carbon peak becomes so weak and predict that the carbon is nearly gone. The chemical bonding state position of silver fibers were also measured using X-ray photoelectron spectroscopy (XPS). There is chemical bonding state position of silver for the sample calcinated at $280^{\circ} \mathrm{C}$ as shown in Figure S10a. The surface chemical bonding of silver fibers is shown in Figure S10b in which the Ag 3d XPS spectrum of silver fibers exhibits two peaks at $368.14 \mathrm{eV}$ and $374.14 \mathrm{eV}$, corresponding to $\mathrm{Ag} 3 \mathrm{~d} 5 / 2$ and $\mathrm{Ag} 3 \mathrm{~d} 3 / 2$ respectively. In comparison with $\mathrm{Ag}^{0}$ (368.25 and $374.25 \mathrm{eV}$ ) and $\mathrm{Ag}_{2} \mathrm{O}(367.70$ and $373.70 \mathrm{eV})$, the peaks are shift down to a negligible extent with respect to $\mathrm{Ag}^{0}$. The slight shift might be due to the presence of the residual $\mathrm{C}, \mathrm{N}$ and $\mathrm{O}$ elements. The color of silver fibers turned from grey to white when the temperature of calcination changed from 250 to 
$280^{\circ} \mathrm{C}$, suggesting that most amorphous carbon on the silver nanofibers surface were removed at a higher temperature.

In order to investigate the crystal structure, silver fibers were characterized by X-ray diffraction pattern which is shown in Figure S5. It is noted that the silver fibers correspond to the normal silver crystal phase and the grain size of synthesized silver nanofibers is about $23 \mathrm{~nm}$ from the full width at half maximum (FWHM) of silver peak at 77.5 degree of $2 \theta$. To further study the micro-structure of silver fibers, transmission electron microscope (TEM) were photographed. As shown in the TEM image of a single silver fiber in Figure S6, we can see that the fiber is combined with compacted silver grains. And in the HRTEM image, the silver grain displays clear lattice fringes, which can be corresponding to the interplanar spacing $d=0.235 \mathrm{~nm}$. However, since the thickness of a single grain is changing according to the position, the selected area electron diffraction (SAED) pattern of insert image in Figure S6 is ununiformed and some points disappeared. On the surface of the silver fiber with insufficient calcination, the existence of the amorphous carbon film with thickness smaller than $5 \mathrm{~nm}$ can be observed as shown in Figure S6. Chemical stability is another important concern for transparent conducting electrodes. Figure S11 shows the change in resistance in silver nanofiber networks upon exposure in air at $80{ }^{\circ} \mathrm{C}$. 


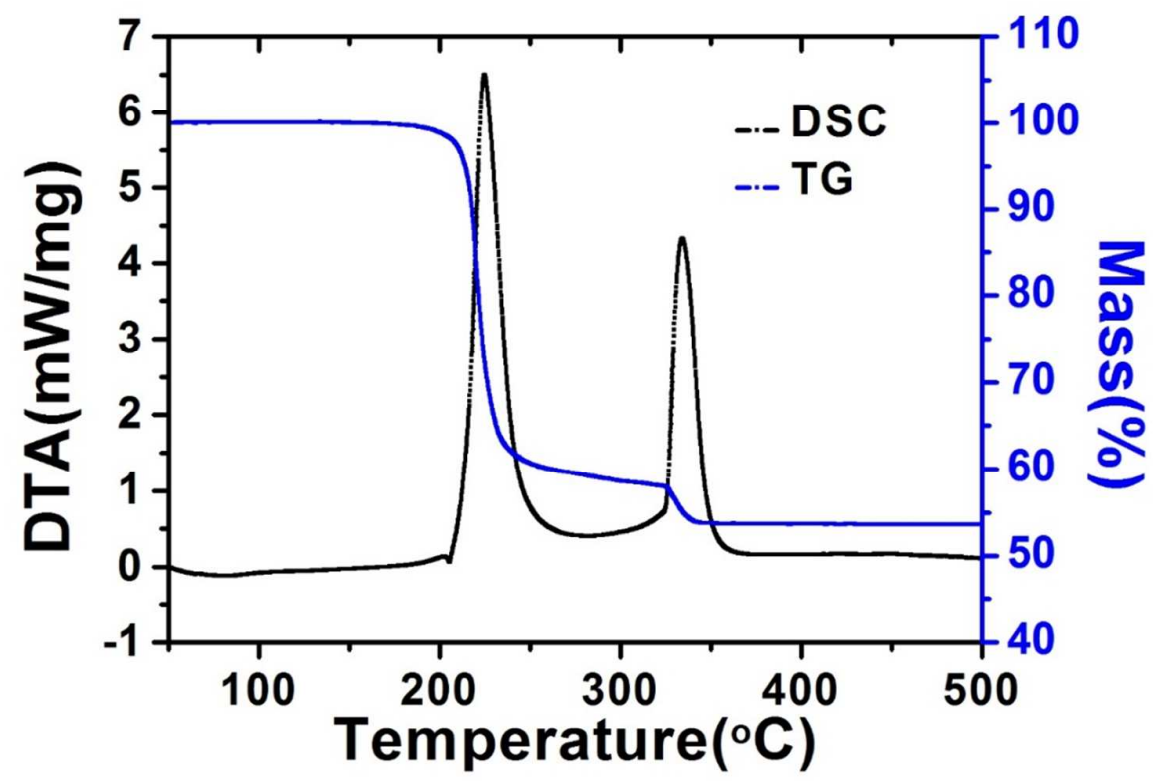

Figure S1. Thermal Gravity Analysis (TGA) and Differential Scanning Calorimetry (DSC) of $\mathrm{PVP} / \mathrm{AgNO}_{3}$ fibers from 50 to $500{ }^{\circ} \mathrm{C}$

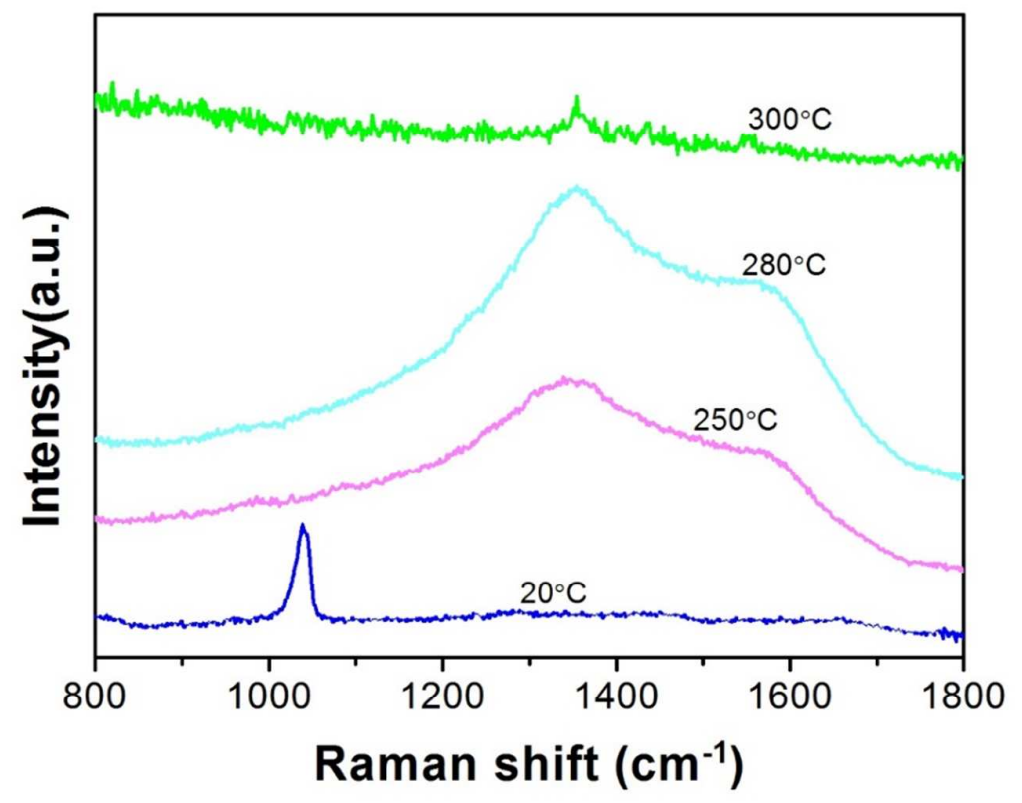

Figure S2. Raman measurement of $\mathrm{PVP} / \mathrm{AgNO}_{3}$ fibers calcinated at different temperature. 

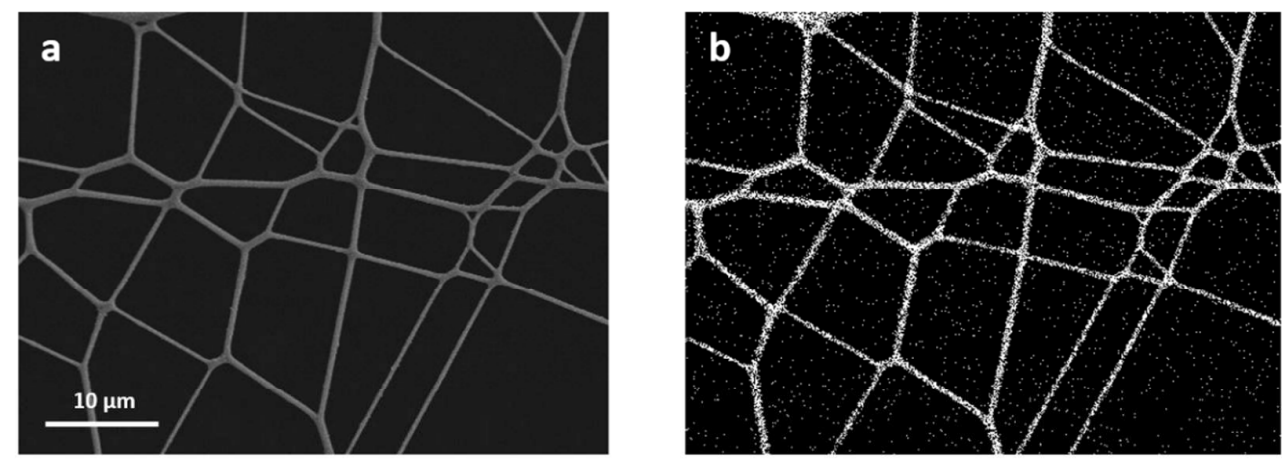

Figure S3. (a) SEM image of silver nanofiber network and (b) Energy Dispersive Spectrometer (EDS) mapping of silver elements in the same area.
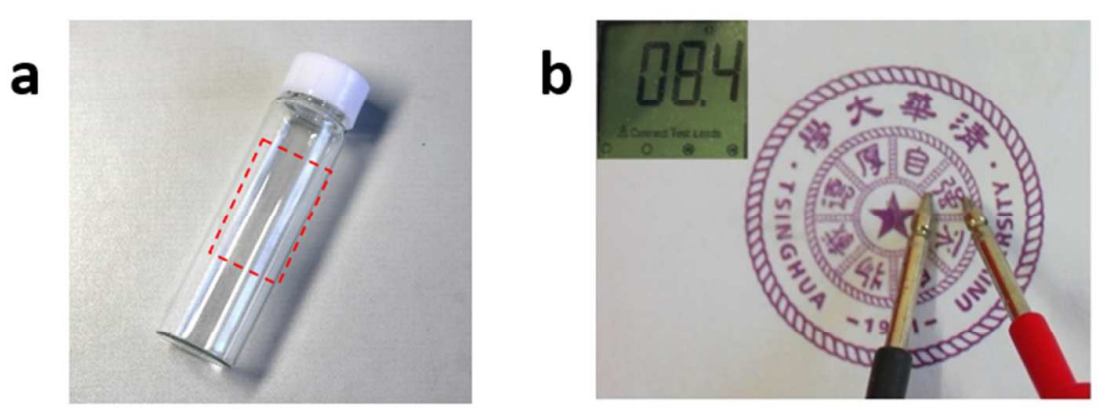

Figure S4. Photograph of silver nanofiber network attached on (a) a glass bottle and (b) paper. 


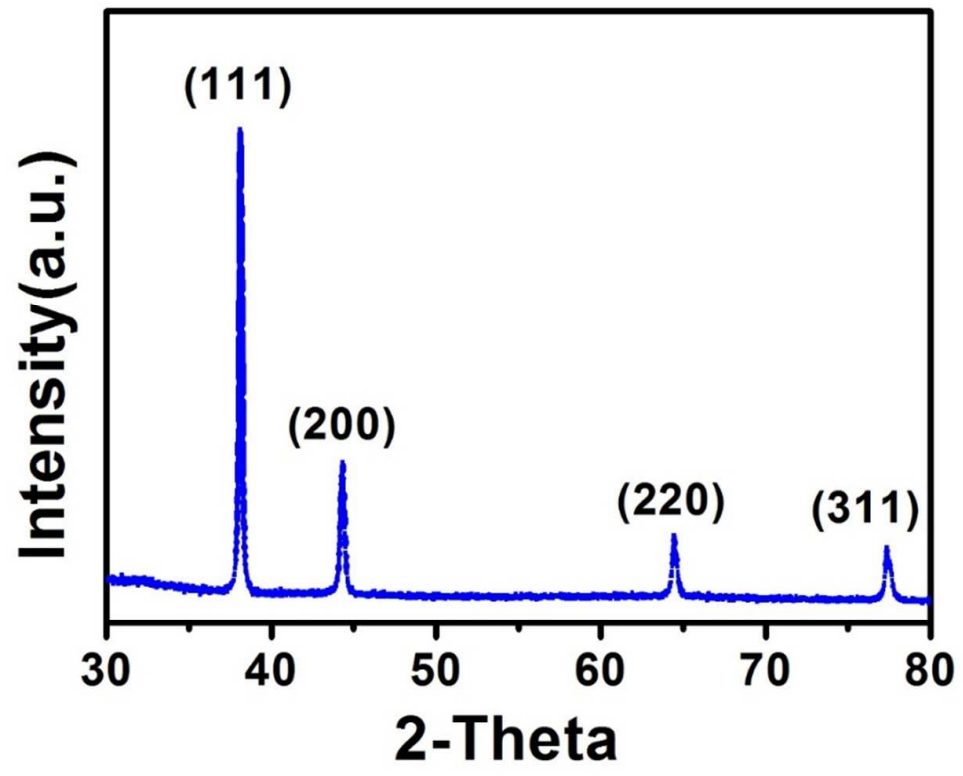

Figure S5. X-ray diffraction pattern of silver nanofibers and silver nanofibers is about $23 \mathrm{~nm}$ from the full width at half maximum (FWHM) of silver peak at 77.5 degree of $2 \theta$.
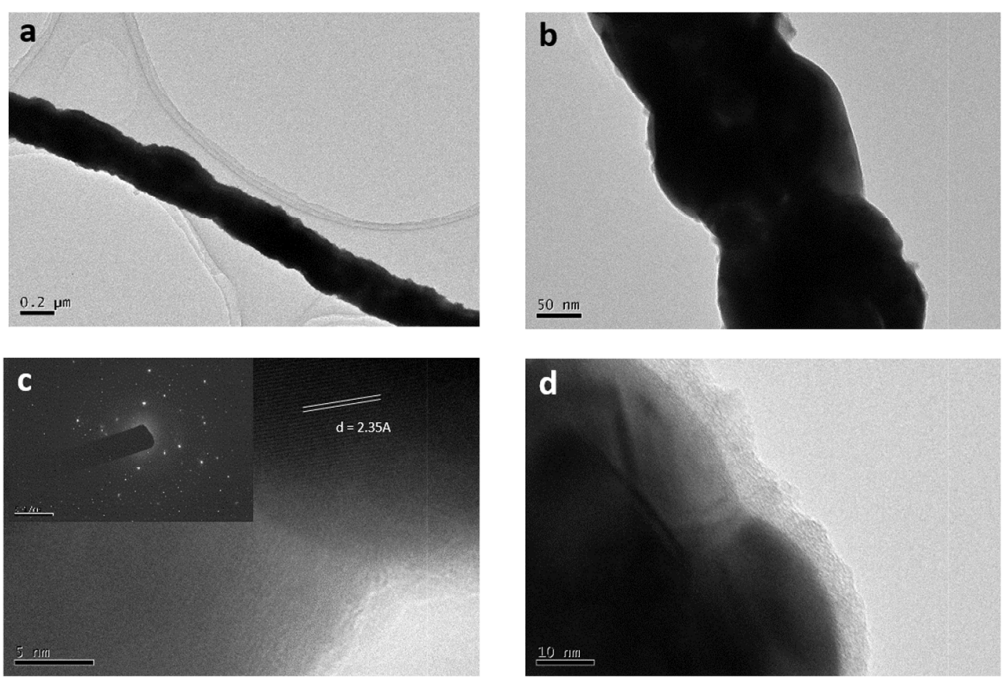

Figure S6. Transmission electron microscope (TEM) measurement of (a) a single silver fiber and (b) the fiber is combined with compacted silver grains. (c) The HRTEM image shows that the silver grain displays clear lattice fringes, corresponding 
to the interplanar spacing $d=0.235 \mathrm{~nm}$. Insert image is the selected area electron diffraction (SAED) pattern. (d) On the surface of the silver fiber with insufficient calcination, the existence of the amorphous carbon film with thickness smaller than 5 nm.

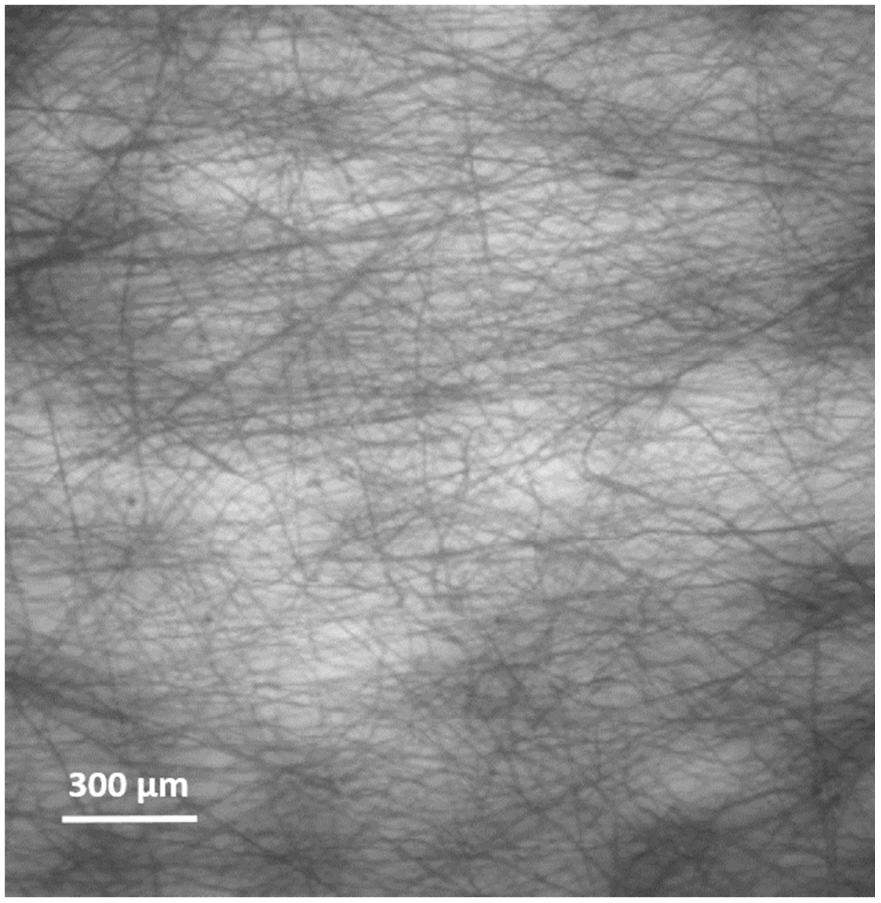

Figure S7. Microscope photograph of silver nanofiber network.
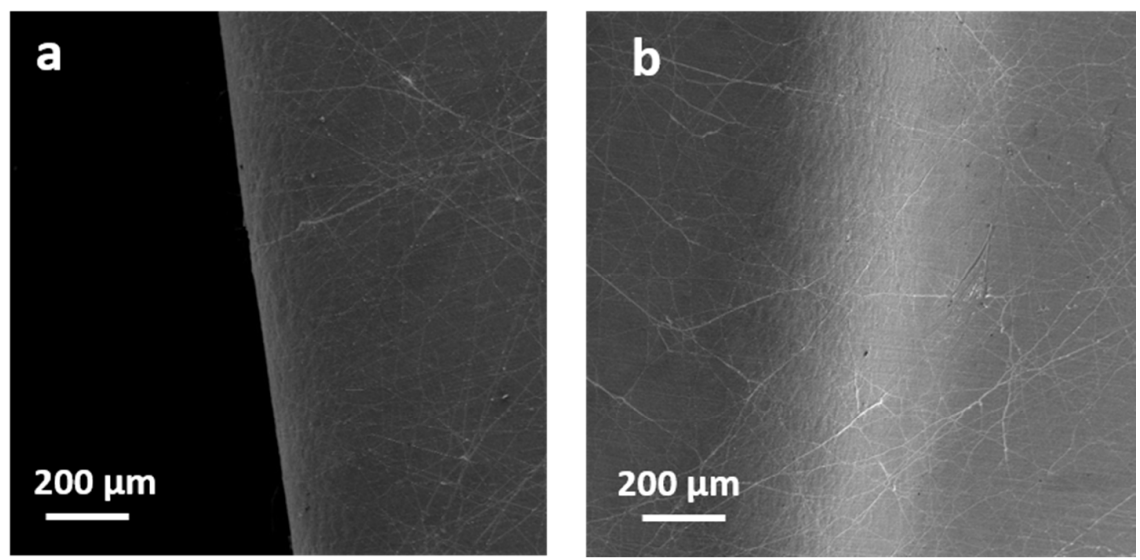

Figure S8. SEM images of silver nanofiber network on aluminum after crushing and 
unfolding. 

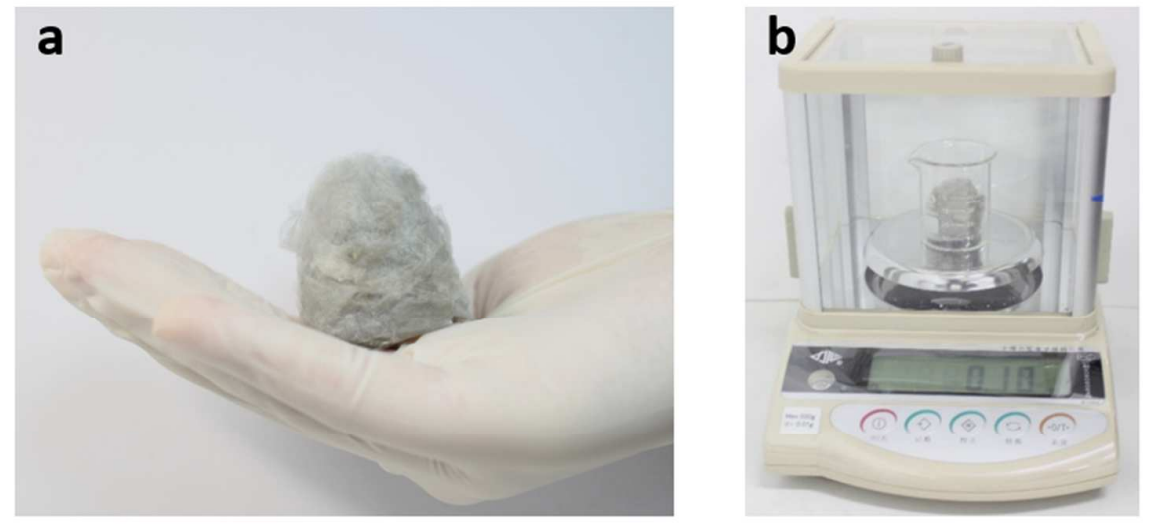

Figure S9. Photograph of (a) silver sponge and (b) weighting measurement.
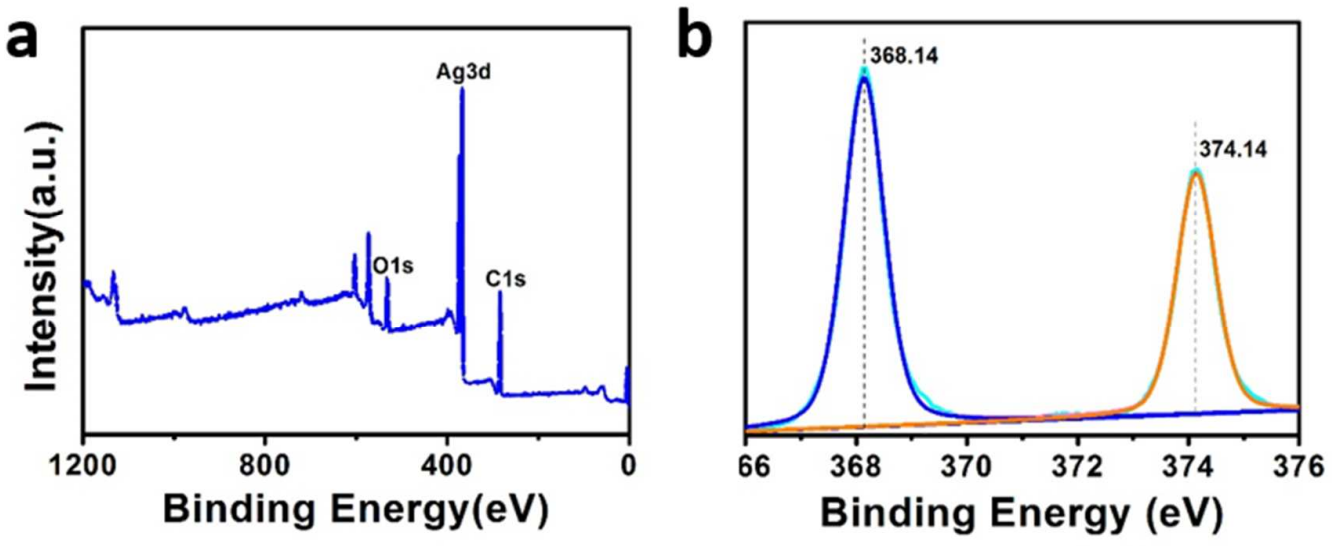

Figure S10. X-ray photoelectron spectroscopy (XPS) measurement of (a) silver nanofibers calcinated at $280{ }^{\circ} \mathrm{C}$; (b) the XPS peak of silver element of AgNF calcinated at $280{ }^{\circ} \mathrm{C}$. 


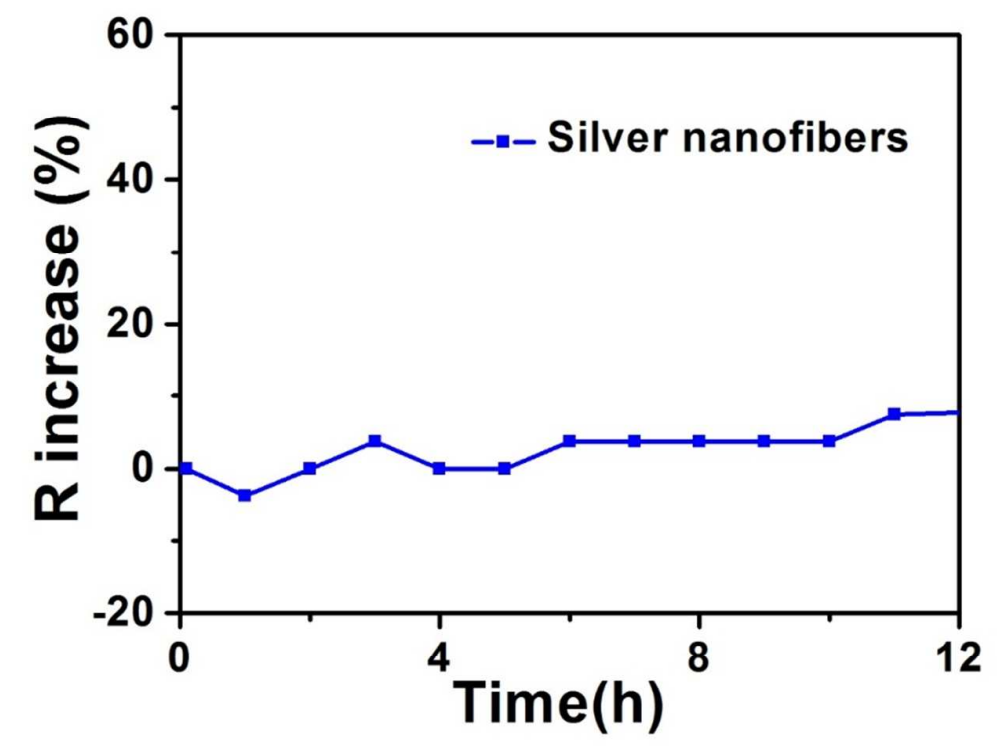

Figure S11. The change of resistance in silver nanofiber networks upon exposure at $80{ }^{\circ} \mathrm{C}$.
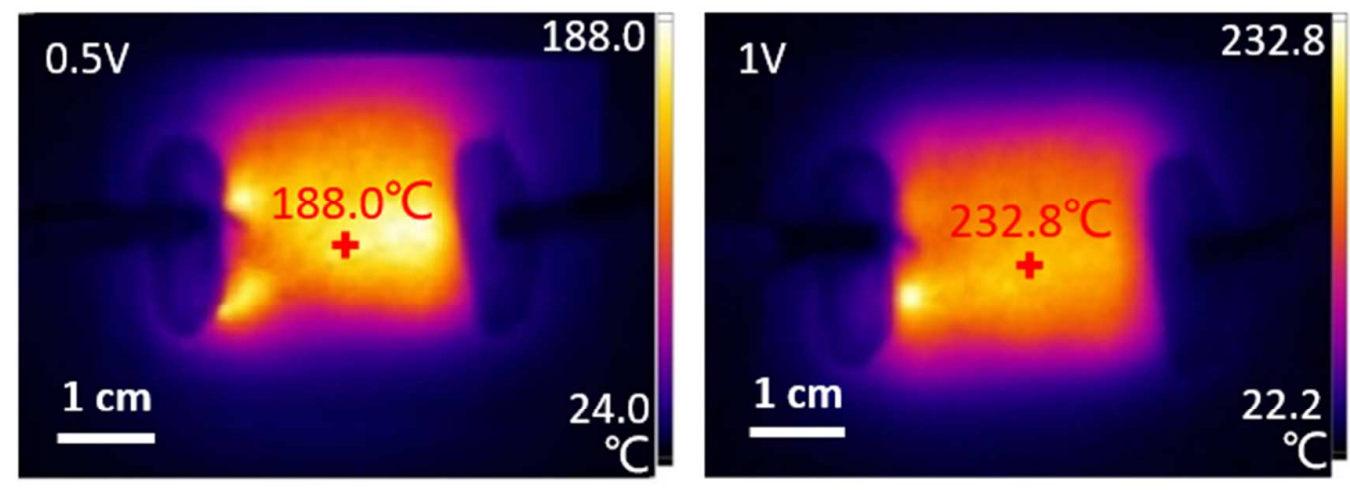

Figure S12. Infrared thermal image of representative steady-state AgNF network with a sheet resistance of $10 \Omega \mathrm{sq}^{-1}$ and which is powered by an applied voltage of $0.5 \mathrm{~V}$ and $1 \mathrm{~V}$. 


\section{Supporting Videos}

Video S1. Crushing experiment of silver nanofiber networks.

Video S2. Pressing measurement of silver sponge. 